\title{
Does insecticide resistance alone account for the low genetic variability of asexually reproducing populations of the peach-potato aphid Myzus persicae?
}

\author{
T Zamoum ${ }^{1}$, J-C Simon ${ }^{2}$, D Crochard ${ }^{1}$, Y Ballanger ${ }^{3}$, L Lapchin ${ }^{1}$, F Vanlerberghe-Masutti ${ }^{1}$ \\ and T Guillemaud ${ }^{1}$ \\ ${ }^{1}$ Equipe 'Biologie des Populations en Interaction', UMR 1112, INRA Antibes 06606, France; ${ }^{2}$ Equipe 'Biologie et génétique des \\ Populations d'insectes' UMR BIO3P, INRA B.P. 29, F-35653 Le Rheu Cedex, France; '3ervice Innovations, CETIOM, Thiverval \\ Grignon 78850, France
}

\begin{abstract}
The typical life cycle of aphids includes several parthenogenetic generations and a single sexual generation (cyclical parthenogenesis), but some species or populations are totally asexual (obligate parthenogenesis). Genetic variability is generally low in these asexually reproducing populations, that is, few genotypes are spread over large geographic areas. Both genetic drift and natural selection are often invoked to account for this low genetic variability. The peachpotato aphid, Myzus persicae, which encompasses both cyclical and obligate parthenogens, has developed several insecticide resistance mechanisms as a consequence of intense insecticide use since the 1950s. We collected asexually reproducing $M$. persicae from oilseed rape and examined genetic variability at eight microsatellite loci and three insecticide resistance genes to determine whether their
\end{abstract}

genetic structure was driven by drift and/or selection. We identified only 16 multilocus microsatellite genotypes among 255 individuals. One clone, which combined two insecticide resistance mechanisms, was frequently detected in all populations whatever their location over a large geographical area (the northern half of France). These unexpected findings suggest that drift is not the unique cause of this low variability. Instead, the intensification of both insecticide treatments and oilseed rape cultivation may have favored a few genotypes. Thus, we propose that selective pressures resulting from human activities have considerably modified the genetic structure of $M$. persicae populations in northern France in a relatively short period of time.

Heredity (2005) 94, 630-639. doi:10.1038/sj.hdy.6800673 Published online 4 May 2005

Keywords: drift; insecticide resistance; $k d r$, overproduced esterases; AchE; microevolution

\section{Introduction}

Like many aphid species, the peach-potato aphid Myzus persicae shows an extreme variation in reproductive modes, from cyclical to obligate parthenogenesis (Blackman, 1972; Vorburger et al, 2003). The annual life cycle of M. persicae includes cyclical parthenogenesis for several generations on herbaceous plants and one sexual generation on peach trees during the winter. By contrast, obligate parthenogenetic aphids have lost the ability to reproduce sexually and remain on herbaceous plants all year long, although they still retain the ability to produce some males (Blackman, 1972).

By using hypervariable microsatellite markers or ITS fingerprints, several studies have shown that asexually reproducing populations of $M$. persicae sampled on herbaceous plants display a low genotypic variability, both within and among populations at a regional scale (Fenton et al, 1998; Terradot et al, 1999; Guillemaud et al,

Correspondence: T Guillemaud, Equipe 'Biologie des Populations en Interaction', UMR 1112, INRA. 400 route des Chappes BP 163, F-06903 Sophia Antipolis, France. E-mail: guillem@antibes.inra.fr

Received 2 June 2004; accepted 14 February 2005; published online 4 May 2005 2003b; Vorburger et al, 2003). These analyses of European and Australian populations also revealed that some clonal genotypes could be repeatedly found in geographically distant (more than $100 \mathrm{~km}$ apart) populations.

What could be the causes of this low genetic variability? Does it result from selective factors favoring a few genotypes or from stochastic factors randomly acting on all genotypes during the asexual phase of the life cycle. Both drift and directional selection, in combination with clonal reproduction, could explain the coexistence of the limited number of clonal genotypes within populations (Balloux et al, 2003; Bengtsson, 2003). However, the low variability observed at a larger scale (within and among populations) is probably not the result of drift alone, because, being a random process, it would result in different genotypes becoming frequent in different populations (Wright, 1969; Bengtsson, 2003). Recent anthropogenic selection, such as insecticide treatment, is likely to explain the low genetic variability observed at a large scale. In insects, pesticide resistance has often evolved from the recent appearance and spread of single (or a few) adaptive genes, which are selected for over large geographical areas (e.g. Guillemaud et al, 1996; Daborn et al, 2002). 
$M$. persicae has evolved at least four insecticide resistance mechanisms in the field: (1) The overproduction of detoxifying esterases, either E4 or FE4, and conferring resistance to organophosphates (OP) and pyrethroids. (2) The modification of the acetylcholinesterase (AChE), the target of OPs and carbamates, providing resistance against dimethyl carbamate insecticides (Moores and Devonshire, 2000). (3) The $k d r$ mutation of the voltage-gated sodium channel (Martinez-Torres et al, 1999), which is the target of pyrethroids. (4) The $r d l$ mutation of the GABA receptor (Anthony et al, 1998), which is the target of cyclodiene insecticides.

The recent acquisition of insecticide resistance genes selected for in large geographical areas, combined with the absence or rarity of sexual recombination, could have led to the low genetic variability observed in $M$. persicae at a wide geographical scale. The comparison of populations from a large geographic area can be used to test this last hypothesis. Moreover, the analysis of the genetic variability of the putatively selected genes would directly address the selective hypothesis.

We adopted this approach by studying the genetic variability of French populations of $M$. persicae collected on oilseed rape (Brassica napus, known as canola in the USA) using eight microsatellite markers and three insecticide resistance genes.

\section{Materials and methods}

\section{Aphid samples}

A total of 22 batches of 13-54 wingless parthenogenetic females of $M$. persicae were collected from oilseed rape in France between 19 October and 16 November 2001 (see Table 1 and Figure 1). The sampling period corresponds to the beginning of the oilseed rape cropping season in France. Only one aphid per plant was collected to avoid repeated sampling of aphids from the same colony, and

Table 1 Characteristics of the samples of Myzus persicae

\begin{tabular}{llrrrr}
\hline Pop & Location & Date & Longitude & Latitude & $\mathrm{n}$ \\
\hline $\mathrm{Pa}$ & Payns & $16 / 11 / 01$ & $03^{\circ} 59^{\prime}$ & $48^{\circ} 23^{\prime}$ & 13 \\
$\mathrm{Ba}$ & Bazanville & $07 / 11 / 01$ & $-00^{\circ} 35^{\prime}$ & $49^{\circ} 18^{\prime}$ & 33 \\
Su_1 & Surgère & $19 / 10 / 01$ & $-00^{\circ} 45^{\prime}$ & $46^{\circ} 07^{\prime}$ & 39 \\
Su_2 & Surgère & $22 / 10 / 01$ & $-00^{\circ} 45^{\prime}$ & & 36 \\
Su_3 & Surgère & $22 / 10 / 01$ & $-00^{\circ} 45^{\prime}$ & & 60 \\
Su_4 & Surgère & $24 / 10 / 01$ & $-00^{\circ} 45^{\prime}$ & & 30 \\
Su_5 & Surgère & $24 / 10 / 01$ & $-00^{\circ} 45^{\prime}$ & & 18 \\
Su_6 & Surgère & $25 / 10 / 01$ & $-00^{\circ} 45^{\prime}$ & & 28 \\
Su_7 & Surgère & $25 / 10 / 01$ & $-00^{\circ} 45^{\prime}$ & & 21 \\
Su_8 & Surgère & $25 / 10 / 01$ & $-00^{\circ} 45^{\prime}$ & & 13 \\
Su_9 & Surgère & $30 / 10 / 01$ & $-00^{\circ} 45^{\prime}$ & & 25 \\
Ch_1 & Chaux & $29 / 10 / 01$ & $04^{\circ} 54^{\prime}$ & $47^{\circ} 08^{\prime}$ & 34 \\
Ch_2 & Chaux & $29 / 10 / 01$ & $04^{\circ} 54^{\prime}$ & & 30 \\
Ch_3 & Chaux & $29 / 10 / 01$ & $04^{\circ} 54^{\prime}$ & & 25 \\
Ch_4 & Chaux & $29 / 10 / 01$ & $04^{\circ} 54^{\prime}$ & & 19 \\
Co & Comblanchien & $11 / 11 / 01$ & $04^{\circ} 55^{\prime}$ & $47^{\circ} 06^{\prime}$ & 35 \\
Fr & Franxault & $11 / 11 / 01$ & $05^{\circ} 17^{\prime}$ & $47^{\circ} 03^{\prime}$ & 29 \\
Le & Lesdains & $24 / 10 / 01$ & $03^{\circ} 16^{\prime}$ & $50^{\circ} 06^{\prime}$ & 23 \\
SH & St Hilaire & $24 / 10 / 01$ & $03^{\circ} 25^{\prime}$ & $50^{\circ} 11^{\prime}$ & 19 \\
SG_1 & St Germain/Eaulnes & $07 / 11 / 01$ & $01^{\circ} 30^{\prime}$ & $49^{\circ} 46^{\prime}$ & 21 \\
SG_2 & St Germain/Eaulnes & $07 / 11 / 01$ & $01^{\circ} 30^{\prime}$ & & 27 \\
Gr & Grignon & $23 / 10 / 01$ & $01^{\circ} 55^{\prime}$ & $48^{\circ} 51^{\prime}$ & 47 \\
\hline
\end{tabular}

Pop is the name of the sample, and $n$ is the number of sampled aphids. See text for details.

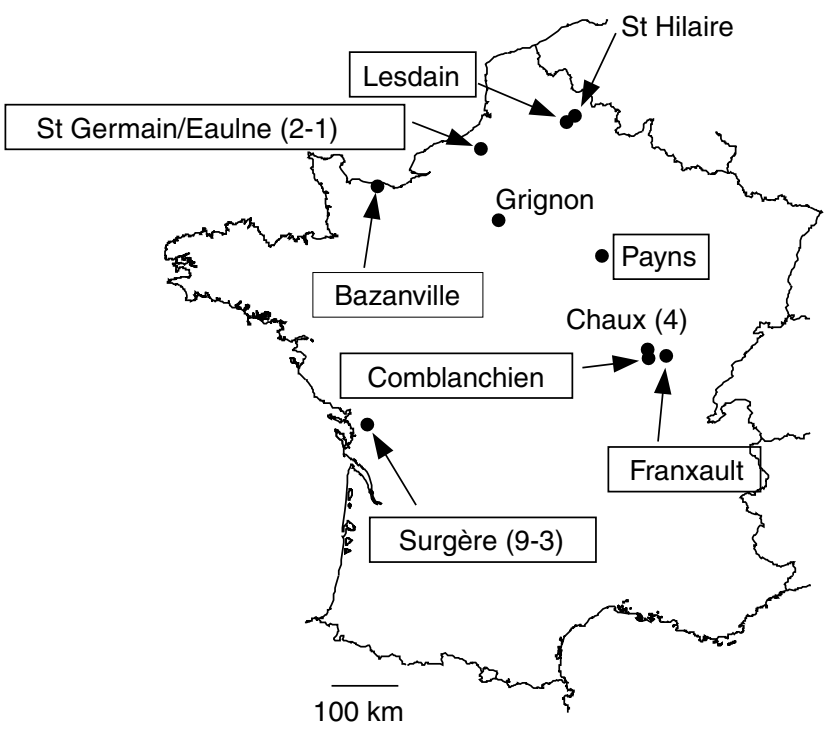

Figure 1 Geographic locations of the $M$. persicae sampling sites Boxed names are sites from which samples were used in the microsatellite analysis. When more than one field was sampled per locality, the first number in brackets corresponds to the number of fields sampled and the second number refers to the number of samples that were used in the microsatellite study.

between 13 and 54 plants were randomly chosen in each field. The aphids were stored at $-80^{\circ} \mathrm{C}$ until analysis. In three locations, samples were collected in October 2001 from both insecticide-treated and untreated fields. In each case, the insecticide was alphamethrin, a pyrethroid insecticide (see Table 2 for details).

Six reference clones described in Guillemaud et al (2003a) were also analyzed: SUS, an insecticide-susceptible clone derived from US1L (Devonshire and Sawicki, 1979); COL and LYON, R2 and R3 clones, respectively, which both overproduce the FE4 esterase (aphids are classified as either S (susceptible), R1, R2 or R3, depending on the levels of esterase overproduction and resistance (Devonshire and Field, 1991)), and are both $k d r / k d s$ and Ace.S/Ace.S (see below for resistance mechanism determination); $\mathrm{AV}$, an R3-FE4, $k d r / k d r$ and Ace.S/ Ace.S clone; BIA, an R3-FE4, $k d s / k d s$ and Ace.R/Ace.S clone; and Mp37/6, an R2-FE4, $k d r / k d r$ and Ace.R/Ace.R clone.

\section{Resistance gene characterization}

For each of the 625 aphids sampled, three insecticide resistance mechanisms were analyzed: the class of esterase overproduction and the corresponding esterase allele, the presence of the $k d r$ mutation and the modified AChE. The $r d l$ mutation was not considered because cyclodiene insecticides are not commonly used against aphids in Europe.

Single aphids were used both for DNA analysis and biochemical assays as described in Guillemaud et al (2003a). Aphids were homogenized in $100 \mu \mathrm{l}$ of $20 \mathrm{mM}$ phosphate buffer $\mathrm{pH} 6.5,0.1 \%$ Triton X-100 (PB-triton) on ice. One-third of the homogenate was used for DNA extraction (Sunnucks and Hales, 1996). The rest of the homogenate was centrifuged for $10 \mathrm{~min}$ at $10000 \mathrm{~g}$. The supernatant was carefully transferred to a new $1.5 \mathrm{ml}$ tube and stored at $-80^{\circ} \mathrm{C}$ until biochemical analysis. 
Table 2 Insecticide resistance phenotype (esterase) and genotypic frequencies (to two decimal places) in samples of $M$. persicae in 2000

\begin{tabular}{|c|c|c|c|c|c|c|c|c|c|c|c|c|c|c|c|c|c|c|}
\hline \multirow[t]{2}{*}{ Pop } & \multirow[t]{2}{*}{$T$} & \multicolumn{4}{|c|}{$\mathrm{kdr}$} & \multicolumn{4}{|c|}{ Esterase overproduction } & \multicolumn{5}{|c|}{ Combination of esterase alleles } & \multicolumn{4}{|c|}{ AChE } \\
\hline & & $\mathrm{n}$ & ss & rs & $r r$ & $\mathrm{n}$ & ss & $R 1-R 2$ & R3 & $\mathrm{n}$ & $E 4$ & FE4 & $E 4 / F E 4$ & $s$ & $\mathrm{n}$ & ss & $r s$ & $r r$ \\
\hline $\mathrm{Pa}$ & & 13 & 0 & 1 & 0 & 12 & 0 & 0.83 & 0.17 & 13 & 0 & 1 & 0 & 0 & 13 & 1 & 0 & 0 \\
\hline Ba & & 33 & 0.06 & 0.94 & 0 & 33 & 0 & 0.58 & 0.42 & 31 & 0.06 & 0.94 & 0 & 0 & 33 & 0.97 & 0.03 & 0 \\
\hline Su_1 & & 39 & 0 & 1 & 0 & 39 & 0 & 0.97 & 0.03 & 37 & 0 & 1 & 0 & 0 & 39 & 1 & 0 & 0 \\
\hline $\mathrm{Su}_{2}{ }_{2}$ & & 35 & 0.29 & 0.66 & 0.06 & 36 & 0 & 1 & 0 & 36 & 0.03 & 0.97 & 0 & 0 & 36 & 0.97 & 0.03 & 0 \\
\hline $\mathrm{Su}_{3} 3$ & & 60 & 0 & 0.95 & 0.05 & 30 & 0 & 0.93 & 0.07 & 57 & 0 & 1 & 0 & 0 & 60 & 0.97 & 0.03 & 0 \\
\hline $\mathrm{Su}_{-}^{-} 4$ & & 30 & 0 & 1 & 0 & 29 & 0 & 0.97 & 0.03 & 30 & 0 & 1 & 0 & 0 & 28 & 1 & 0 & 0 \\
\hline $\mathrm{Su}_{-5}$ & & 18 & 0.11 & 0.89 & 0 & 18 & 0 & 1 & 0 & 18 & 0 & 1 & 0 & 0 & 18 & 1 & 0 & 0 \\
\hline Su_6 & & 27 & 0 & 0.96 & 0.04 & 28 & 0 & 0.71 & 0.29 & 28 & 0 & 1 & 0 & 0 & 27 & 1 & 0 & 0 \\
\hline $\mathrm{Su}_{-}^{-7}$ & & 20 & 0.05 & 0.95 & 0 & 21 & 0 & 0.81 & 0.19 & 21 & 0.05 & 0.95 & 0 & 0 & 21 & 1 & 0 & 0 \\
\hline $\mathrm{Su}_{-} 8$ & & 10 & 0 & 1 & 0 & 13 & 0 & 1 & 0 & 10 & 0 & 0.9 & 0 & 0.1 & 13 & 1 & 0 & 0 \\
\hline $\mathrm{Su}_{-}$9 & & 22 & 0.23 & 0.73 & 0.05 & 25 & 0 & 0.76 & 0.24 & 20 & 0.05 & 0.95 & 0 & 0 & 25 & 1 & 0 & 0 \\
\hline $\mathrm{Co}^{-}$ & & 35 & 0.20 & 0.80 & 0 & 35 & 0 & 0.63 & 0.37 & 35 & 0.03 & 0.97 & 0 & 0 & 34 & 1 & 0 & 0 \\
\hline $\mathrm{Fr}$ & & 26 & 0.08 & 0.92 & 0 & 29 & 0 & 0.52 & 0.48 & 27 & 0 & 1 & 0 & 0 & 29 & 1 & 0 & 0 \\
\hline Le & nt & 22 & 0.18 & 0.73 & 0.09 & 23 & 0 & 0.96 & 0.04 & 20 & 0 & 1 & 0 & 0 & 23 & 0.91 & 0.09 & 0 \\
\hline SH & $\mathrm{t}$ & 18 & 0.11 & 0.89 & 0 & 19 & 0 & 0.26 & 0.74 & 19 & 0 & 1 & 0 & 0 & 19 & 0.89 & 0.11 & 0 \\
\hline Ch_1 & $t$ & 34 & 0 & 0.97 & 0.03 & 32 & 0 & 1 & 0 & 33 & 0 & 1 & 0 & 0 & 34 & 1 & 0 & 0 \\
\hline Ch_2 & nt & 30 & 0.03 & 0.93 & 0.03 & 30 & 0 & 1 & 0 & 30 & 0 & 1 & 0 & 0 & 29 & 1 & 0 & 0 \\
\hline Ch_3 & $\mathrm{nt}$ & 22 & 0 & 0.91 & 0.09 & 25 & 0 & 0.88 & 0.12 & 25 & 0 & 1 & 0 & 0 & 25 & 1 & 0 & 0 \\
\hline $\mathrm{Ch}_{-} 4$ & $\mathrm{t}$ & 18 & 0 & 1 & 0 & 19 & 0 & 1 & 0 & 19 & 0 & 1 & 0 & 0 & 19 & 1 & 0 & 0 \\
\hline SG_1 & $\mathrm{nt}$ & 20 & 0.1 & 0.9 & 0 & 0 & - & - & - & 21 & 0.05 & 0.8 & 0.1 & 0.05 & 21 & 1 & 0 & 0 \\
\hline $\mathrm{SG}_{2} 2$ & $t$ & 23 & 0.39 & 0.61 & 0 & 24 & 0 & 1 & 0 & 23 & 0.3 & 0.7 & 0 & 0 & 25 & 1 & 0 & 0 \\
\hline Gr & & 47 & 0.11 & 0.89 & 0 & 47 & 0 & 1 & 0 & 46 & 0.09 & 0.91 & 0 & 0 & 47 & 1 & 0 & 0 \\
\hline Total & & 602 & 0.09 & 0.89 & 0.02 & 567 & 0 & 0.85 & 0.15 & 599 & 0.03 & 0.97 & 0 & 0 & 618 & 0.99 & 0.01 & 0 \\
\hline
\end{tabular}

For each resistance mechanism, the number of individuals analyzed, $n$, is given. The column ' $\mathrm{T}$ ' indicates if the fields were treated ( $\mathrm{t}$ ) or not (nt) with pyrethroid insecticides before sampling.

Esterase overproduction: The presence of the E4 and FE4 alleles at esterase genes in individual aphids was determined using a modified version (Guillemaud et al, 2003a) of the PCR-RFLP method described by Field et al (1996). Genotypes could not be determined using this method because the esterase loci can be located at several different chromosomal sites (Blackman et al, 1995).

The level of E4 and/or FE4 esterase overproduction was estimated for each aphid by using the microplate total-esterase assay described by Devonshire et al (1992). This method can distinguish between three classes of aphids: susceptible, R1/R2 and R3 (Devonshire and Field, 1991).

Ace genotype determination: A fluorometric method was used to determine the genotype at the AChE locus, Ace (Moores and Devonshire, 2000; Guillemaud et al, 2003a). Only the two alleles described by Moores et al (1994) were considered: Ace.S (the allele encoding the susceptible $\mathrm{AChE}$ ) and Ace.R (the allele encoding the modified, resistant AChE). Each individual was classified as either Ace.S/Ace.S, Ace.R/Ace.S or Ace.R/ Ace.R.

$K d r$ determination: Pyrethroid resistance is correlated with the presence of the $k d r$ mutation, that is, the presence of a T ( $k d r$ allele) instead of C ( $k d s$ allele) at position 322 of the partial cDNA sequence of the para-like gene of M. persicae (Martinez-Torres et al, 1999). A PCR amplification of specific alleles (PASA) procedure (Sommer et al, 1992) was used to determine individual genotypes ( $k d s / k d s, k d r / k d s$ and $k d r / k d r)$ at this position as described by Guillemaud et al (2003a). In the following, the para-like gene will be referred to as $k d r$ gene.

\section{Microsatellite analysis}

Nine of the 22 samples were subjected to microsatellite analysis to investigate the genotypic variability of $M$. persicae collected on oilseed rape over the geographic range considered for the insecticide resistance genes study: $\mathrm{Le}, \mathrm{Ba}, \mathrm{Co}, \mathrm{Fr}, \mathrm{Pa}, \mathrm{Su} \_1, \mathrm{Su} \_2, \mathrm{Su} \_3$ and SG_2 (see Figure 1). Each aphid was genotyped using eight microsatellite loci: five (Myz25, M37, M40, M55 and M62) were isolated from M. percicae and three (S16b, S17b and S23) from Sitobion miscanthi (Takahashi) (Wilson et al, 2004).

The microsatellites were amplified according to Sloane et al (2001) using the PMS1 PCR program. Microsatellite products were then loaded onto an $8 \%$ nondenaturing polyacrylamide gel and visualized by ethidium bromide staining as described by Vanlerberghe-Masutti et al (1999). Each individual was described by its multilocus genotype (MLG): the allelic combination at the eight microsatellite loci. The observed MLGs were then carefully scored by using an automatic sequencer (ABI Prism 3100, Applied Biosystem) and reference DNAs from P. Sunnucks (La Trobe University, Australia).

\section{Analysis of genetic data}

Genetic variability: Allele frequencies, number of alleles and heterozygosity were calculated using the GENEPOP (ver. 3.3) software (Raymond and Rousset, 1995b). Hardy-Weinberg (H-W) equilibrium and linkage equilibrium are not expected if the samples display a clonal structure because of repeated parthenogenetic generations. We therefore tested for these equilibriums to 
evaluate the relative importance of the successive parthenogenetic generations and of the putative sexual reproduction in shaping the population genetic structure. Linkage disequilibrium (LD) between loci within each population and departure from $\mathrm{H}-\mathrm{W}$ equilibrium at each locus were tested using the pseudo-exact test procedures in GENEPOP 3.3. Note that the LD test does not assume HW equilibrium. We built a neighborjoining tree based on allele shared distance (DAS) using Population (version 1.2.23 from http:/ / www.cnrs-gif.fr / pge) to investigate relationships between MLGs.

Between-sample analysis: Differences in the frequencies of insecticide resistance mechanisms between populations were analyzed by testing for genic and genotypic differentiation (pseudo-exact test described in Raymond and Rousset, 1995a).

To take into account departures from $\mathrm{H}-\mathrm{W}$ and linkage equilibria, the following statistical treatments were applied to MLGs. The spatial genetic structure revealed by the microsatellites was analyzed by estimating $F_{\mathrm{ST}}$ parameters (Weir and Cockerham, 1984) and by testing for MLG frequencies homogeneity using the Struc program in the GENEPOP 3.3 package. The use of $F_{\mathrm{ST}}$ to study a species with a mixture of cyclical and obligate parthenogens may be problematic. Indeed, $F_{\mathrm{ST}}$ can be hard to interpret in asexually reproducing populations where it does not necessarily reflect migration/drift equilibrium because of selection at loci which have not been scored. However, we chose this genetic distance parameter to enable comparisons with other published population genetics studies on aphids (eg Delmotte et al, 2002; Guillemaud et al, 2003b). The Isolde program from the GENEPOP package was used to test for an MLG isolation by distance model. This program performs Mantel tests of independence between the matrix of genetic distance and that of geographic distance.

General statistical methods: Most of the statistical genetics analyses described here were performed on the whole data set because (1) we had no a priori information about the reproductive mode of $M$. persicae populations (ie cyclical vs obligate parthenogenesis) and (2) very few MLGs were found to consider only one individual per MLG (and per population for the geographic structure analysis). The sequential Bonferroni correction was applied for nonorthogonal tests, that is, pairwise differentiation and LD tests (Sokal and Rohlf, 1995, pp 241-242). In each sample, this correction was also applied to the several (one per locus) H-W equilibrium tests performed (Sokal and Rohlf, 1995, pp 241-242). Overall significance of several independent tests was calculated using Fisher's method (Sokal and Rohlf, 1995, pp 794-797).

\section{Results}

Frequency of insecticide resistance genes in the field The frequencies of the insecticide resistance phenotypes and genotypes are given in Table 2. A significant association was detected between genotypes at the $k d r$ gene and esterase alleles across all populations (combination of the probabilities by the Fisher's method, $P<0.001)$ and within $\mathrm{Gr}$ and SG samples $\left(P<10^{-4}\right.$,
$\mathrm{SE}<10^{-4}$ for each test). This corresponded to an association between the E4 elevated esterase and the $k d s / k d s$ genotype.

The FE4 allele was present in more than $95 \%$ of the aphids sampled from all sites. On the contrary, the allele encoding the E4 esterase was very rare (about 3\% of the aphids). Two individuals displayed both overproduced esterase alleles and two aphids had alleles that did not correspond to either E4 or FE4 according to the PCRRFLP method. These two aphids were classified as R1/ R2. About $85 \%$ of the individuals were classified as R1/ $\mathrm{R} 2$ and $15 \%$ as R3.

Unexpectedly, the modified acetycholinesterase described by Moores et al (1994) was absent from most samples. Only eight aphids were found to be heterozygous for this insensitive AChE. In the polymorphic samples and when the tests could be performed, H-W equilibrium was not rejected $(P>0.05$ in each case).

Among the 625 aphids analyzed, 23 could not be genotyped for the $k d r$ mutation by the PASA method because no PCR products were obtained. In all, $89 \%$ of the individuals were $k d r / k d s$ and $2 \%$ were $k d r / k d r$. Most samples (except Le, Su_2, Su_9 and SG_2) displayed significant heterozygous excess, with $\bar{F}_{\text {IS }}$ estimates ranging from -1 to -0.37 .

When considering the three different resistance mechanisms, 13 classes of aphids were found. One type was particularly frequent: $76 \%$ of the aphids were $k d r / k d s$, Ace.S/Ace.S, R1-R2 and had the elevated esterase FE4. In all, $12 \%$ were $k d r / k d s$, Ace.S/Ace.S, R3 and FE4. All other types of aphids were found at a low frequency. In total, $90 \%$ of the aphids analyzed had both the $k d r$ mutation and an elevated esterase.

Resistance-gene differentiation between populations Differentiation between populations was tested using the genetic information obtained from insecticide resistance genes. Significant differences in the frequencies of esterase overproduction classes and of $k d r$ genotypes were found among populations $\left(P<10^{-5}\right.$ for both resistance mechanisms). We then compared samples treated (SH, Ch 1, Ch 4 and SG 2) or not (Le, Ch 2, Ch_3 and SG_1) with pyrethroid insecticides. Significant differentiation was found in the frequency of esterase overproduction categories between the treated $\mathrm{SH}$ sample and the three untreated samples for which data were available (Le, Ch_2 and Ch_3, $P<2 \times 10^{-4}$ for each comparison). In each case, the insecticide-treated $\mathrm{SH}$ sample contained more R3 and less R1/R2 individuals than the samples from the untreated fields (see Table 2). Genotypic differentiation at the $k d r$ gene was also found between the treated SG_2 sample and the untreated populations $\mathrm{Ch} 22$ and $\mathrm{Ch} 3\left(P=1.5 \times 10^{-3}\right.$, $\mathrm{SE}=4 \times 10^{-4}$; and $P=4 \times 10^{-4}, \mathrm{SE}=10^{-4}$, respectively) However, the treated sample contained more susceptible $k d s / k d s$ individuals (39\%) than the untreated ones (less than 3\%, see Table 2). When considering only groups of populations that were treated or untreated and that were sampled from the same location (SG_1 and SG_2, Le and $\mathrm{SH}$ and the four $\mathrm{Ch}$ samples), no differentiation was found for any of the resistance mechanisms except for the esterase overproduction class between Le and SH. 
Within-sample 'neutral' genetic diversity

Samples from nine fields $\left(\mathrm{Su}_{-}\right.$1, $\mathrm{Su} \_2, \mathrm{Su}$ 3, Le, SG_2, Ba, $\mathrm{Co}$, Fr and $\mathrm{Pa}$ ) were genotyped at the eight microsatellite loci. The genetic markers proved to be polymorphic, with 2-8 alleles per locus. The genotypic diversity was globally low, with three (loci S23 and S16b) to eight (loci Myz25, M37, M40 and M62) genotypes per locus.

A total of 21 pairs of loci out of 28 showed significant LD over all populations $\left(P<10^{-4}\right.$ for each significant comparison). The $\mathrm{H}-\mathrm{W}$ equilibrium was often rejected (Table 4), frequently due to heterozygote excess (ie negative $F_{\text {IS }}$ estimates).

Only 16 MLGs were detected among the 255 individuals (Table 3). One frequent MLG, referred to as G1 hereafter, was found in $86 \%$ of the aphids. The other genotypes were rare and the most frequent of them had a frequency of only $4.6 \%$. Most of these rare MLGs corresponded to single-copy MLGs $(0.4 \%$ in Table 3$)$. It is noteworthy that the occurrence of heterozygote deficiency or excess at a locus depends on whether G1 was heterozygote or homozygote at the locus considered. The neighbor-joining tree based on DAS distances (Figure 2) revealed that, with the exception of G14, which differs from G1 by only two alleles, none of the detected MLGs were closely related to G1, as at least five alleles differentiated G1 from the other genotypes.

When considering only one individual per MLG, the $\mathrm{H}-\mathrm{W}$ equilibrium was rejected for loci Myz25, M55 and M62 $(P<0.015, \mathrm{SE}<0.02)$, with $F_{\mathrm{IS}}$ estimates ranging from 0.17 to 0.69 for significant deviations. No significant LD was detected within these 16 individuals $(P>0.05$, $\mathrm{SE}<10^{-2}$ for each pair of loci).

\section{'Neutral' genetic differentiation between samples}

The spatial genetic structure was analyzed by testing the homogeneity of MLG frequencies between samples at various spatial scales. An indication of the genetic differentiation between samples or groups of samples was given by $F_{\mathrm{ST}}$ estimates.

Samples of eastern France (Co, Fr and $\mathrm{Pa}$ ) showed no significant MLG differentiation $\left(P>0.05 ; F_{\mathrm{ST}}=0.008\right)$, and were therefore lumped together in the following analyses of spatial structure. Among western samples, a significant and moderate differentiation $\left(P<10^{-4}\right.$, $\left.\mathrm{SE}<10^{-4}, F_{\mathrm{ST}}=0.04\right)$ was found and was due to the Su2 sample (Table 5) in which $22 \%$ of the aphids displayed the G2 MLG (Table 3). The three samples in northern France were significantly heterogeneous overall
$\left(P<10^{-4}, \mathrm{SE}<10^{-4}, F_{\mathrm{ST}}=0.05\right)$, and in each pair-wise comparison (Table 5).

The seven remaining samples or group of samples were significantly but moderately heterogeneous for their MLG composition $\left(P<10^{-5}, \mathrm{SE}<10^{-5} ; F_{\mathrm{ST}}=0.032\right)$. The same differentiation pattern was obtained when considering the frequency of G1 only (not shown). After correction for nonorthogonal tests, no differentiation was found between eastern and western samples (Table 5). No pattern of isolation by distance was observed between the samples, that is, no significant correlation was found between the matrices of genetic distance $\left(F_{\mathrm{ST}}\right.$, $F_{\mathrm{ST}} /\left(1-F_{\mathrm{ST}}\right)$ or DAS) and of log geographic distance (Mantel test with 10000 permutations, $P>0.05$; see Rousset (2001) for details).

\section{Resistance genes and microsatellite combinations}

We next looked at the correspondence between the microsatellite genotypes and the resistance genes (Table 6). Esterase overproduction phenotypes were not

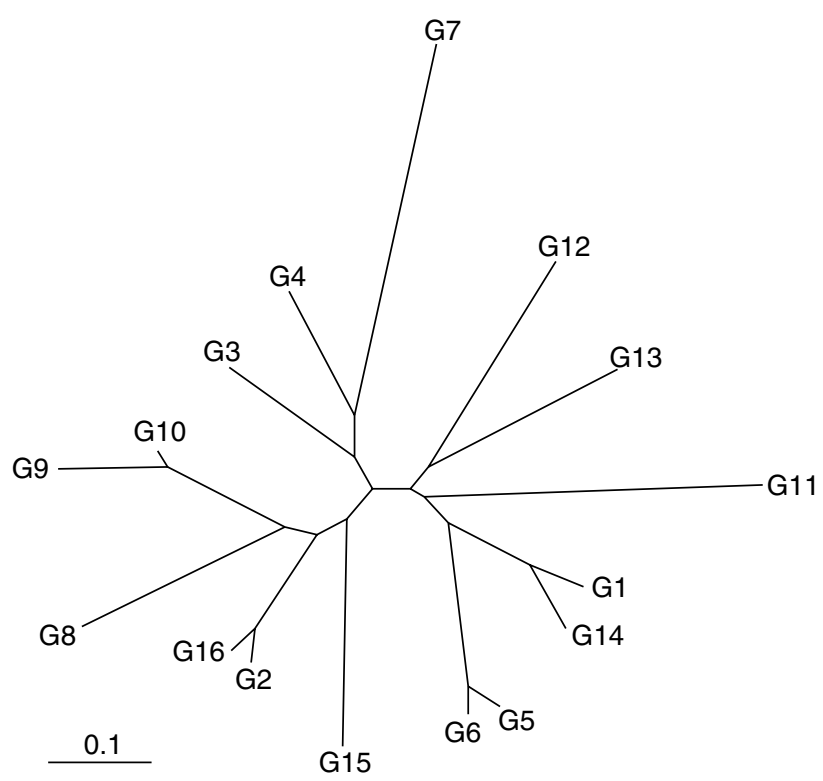

Figure 2 Neighbor-joining tree of the 16 multilocus microsatellite genotypes detected in $M$. persicae sampled from oilseed rape fields of northern France based on the allele shared distance. With eight loci, a 'one allele' distance is $1 / 16=0.0625$.

Table 3 Distribution of the microsatellite multilocus genotypes (in \% to one decimal place) among the samples of Myzus persicae (see Table 5 for details of the genotypes)

\begin{tabular}{|c|c|c|c|c|c|c|c|c|c|c|c|c|c|c|c|c|c|}
\hline Pop & $\mathrm{n}$ & G1 & G2 & G3 & G4 & G5 & G6 & G7 & G8 & G9 & G10 & G11 & G12 & G13 & G14 & G15 & G16 \\
\hline $\mathrm{Su} \_1$ & 39 & 100 & - & - & - & - & - & - & - & - & - & - & - & - & - & - & - \\
\hline Su_3 & 30 & 96.7 & - & - & 3.3 & - & - & - & - & - & - & - & - & - & - & - & - \\
\hline $\mathrm{Su} \_2$ & 36 & 69.4 & 22.2 & - & - & - & - & 2.8 & 2.8 & - & 2.8 & - & - & - & - & - & - \\
\hline Le & 22 & 72.7 & - & - & 4.5 & 4.5 & 4.5 & - & - & 4.5 & - & - & - & 4.5 & - & - & 4.5 \\
\hline SG_2 & 24 & 62.5 & - & 37.5 & - & - & - & - & - & - & - & - & - & - & - & - & - \\
\hline $\mathrm{Ba}$ & 33 & 93.9 & - & 6.1 & - & - & - & - & - & - & - & - & - & - & - & - & - \\
\hline Co & 30 & 80 & 10 & - & - & - & - & - & - & - & - & 3.3 & 3.3 & - & 3.3 & - & - \\
\hline $\mathrm{Fr}$ & 28 & 96.4 & - & - & - & - & - & - & - & - & - & - & - & - & - & 3.6 & - \\
\hline $\mathrm{Pa}$ & 13 & 100 & - & - & - & - & - & - & - & - & - & - & - & - & - & - & - \\
\hline Total & 255 & 85.9 & 4.3 & 4.3 & 0.8 & 0.4 & 0.4 & 0.4 & 0.4 & 0.4 & 0.4 & 0.4 & 0.4 & 0.4 & 0.4 & 0.4 & 0.4 \\
\hline
\end{tabular}


considered because of the possibility of phenotypic variation within genotypes. One category of individual predominated (83.5\% of aphids). This group had the G1 genotype, was heterozygous for the $k d r$ mutation, homozygous for the susceptible form of the AChE and displayed the overproduced esterase FE4. All the other combinations were very rare, with frequencies ranging from 0.4 to $4.6 \%$. Most of the rare combinations (13 out of 20) corresponded to $k d s / k d s$ aphids, but they accounted for no more than 13\% of the total. Apart from the G1 aphids, only four genotypes (and four individuals) had the $k d r$ mutation.

No strict associations could be established between the different kinds of markers because (a) no significant linkage could be detected between microsatellites and insecticide resistance markers when only one individual per MLG (20 individuals) was considered, (b) each resistance mechanism was found in different MLGs and (c) some resistance genes showed variation within microsatellite MLGs (Table 6). Indeed, four different insecticide resistance gene associations were found within G1, comprising all $k d r$ genotypes and both the Ace.S/Ace.S and Ace.R/Ace.S genotypes.

\section{Discussion}

Putative reproductive modes of $M$. persicae genotypes It has been shown that cyclical and obligate parthenogenetic populations of $M$. persicae can coexist at large but also at local geographic scales (Vorburger et al, 2003). We have no direct evidence of whether our sampled aphids come from lineages that are capable of sexuality each year (cyclical parthenogens) or not (obligately parthenogens). Here, M. persicae individuals were sampled in the mid-autumn on herbaceous hosts while they were reproducing parthenogenetically. Most cyclical parthenogens have already switched back at this time of the year to winter hosts, the peach trees, to accomplish sexual reproduction. It is thus probable that our samples contained partially or entirely asexual genotypes.

The analysis revealed frequent $\mathrm{H}-\mathrm{W}$ and linkage disequilibria, that a particular MLG was frequent within populations and had a ubiquitous distribution among geographically distant populations. Can sexual reproduction in the preceding winter account for this peculiar genetic structure? A founder event in which a few sexually produced aphids establish in each field, followed by clonal reproduction, might account for the within-population genetic structure. However, when considering the ubiquitous distribution of G1 among population, it would then be necessary to suppose that G1 appeared independently by recombination at least 9 times in nine distant populations.

Wilson et al (2002) and Guillemaud et al (2003b) showed that sexual $M$. persicae aphids harbor a large microsatellite variability suggesting that the likelihood of obtaining many individuals with the same MLG by sexual recombination in a population is minute. However, it is worth noting that selection could notably increase this likelihood by rapidly increasing the frequency of certain clones during the parthenogenetic phase of their life cycle. Alternatively, we could suppose that a single individual with the G1 MLG was produced by sexual reproduction or by mutation from an obli- 
Table 5 Analysis of genetic population differentiation in M. persicae based on the microsatellite analysis

\begin{tabular}{|c|c|c|c|c|c|c|c|c|c|}
\hline & \multicolumn{3}{|c|}{ Eastern France } & \multicolumn{3}{|c|}{ Western France } & \multicolumn{3}{|c|}{ Northern France } \\
\hline & Co & $\mathrm{Fr}$ & $\mathrm{Pa}$ & $S u \_1$ & $S u \_3$ & $S u \_2$ & Le & $S G_{-} 2$ & $B a$ \\
\hline Co & & 0.01 & 0.01 & & & & & & \\
\hline $\mathrm{Fr}$ & 0.10 & . & 0 & 0.01 & 0.00 & 0.03 & 0.02 & 0.07 & 0.00 \\
\hline $\mathrm{Pa}$ & 0.41 & 1 & & & & & & & \\
\hline Su_1 & & 0.712 & & & 0.00 & 0.06 & 0.05 & 0.10 & 0 \\
\hline $\mathrm{Su}_{-} 3$ & & 0.677 & & 0.438 & 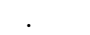 & 0.05 & 0.03 & 0.08 & 0 \\
\hline $\mathrm{Su}_{-} 2$ & & 0.004 & & $<10^{-3}$ & 0.003 & . & 0 & 0.07 & 0.05 \\
\hline Le & & 0.018 & & 0.001 & 0.063 & 0.007 & & 0.05 & 0.03 \\
\hline SG_2 & & $<10^{-3}$ & & $<10^{-3}$ & $<10^{-3}$ & $<10^{-3}$ & 0.002 & 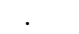 & 0.06 \\
\hline $\mathrm{Ba}$ & & 0.340 & & 0.206 & 0.358 & 0.002 & 0.023 & 0.006 & . \\
\hline
\end{tabular}

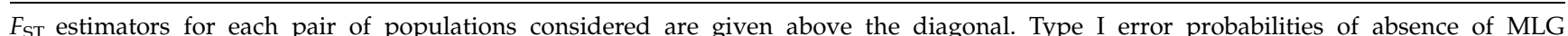
differentiation between populations are given below the diagonal. Bold characters indicated tests that were significant after the Bonferroni correction.

Table 6 Global frequencies of the different types of individuals observed combining the microsatellite data and the insecticide resistance genotypes and phenotypes

\begin{tabular}{|c|c|c|c|c|c|c|c|c|c|c|c|c|}
\hline$G i$ & Myz25 & M37 & M40 & $S 16 b$ & $S 17 b$ & S23 & M62 & M55 & $\mathrm{kdr}$ & $A C h E$ & Est. allele & freq \\
\hline G1 & $119 / 119$ & $155 / 163$ & $125 / 125$ & $196 / 196$ & $168 / 168$ & $115 / 115$ & $131 / 135$ & $119 / 127$ & rs & ss & FE4 & 83.5 \\
\hline - & & & & & & & & & $\mathrm{rr}$ & ss & FE4 & 0.4 \\
\hline - & & & & & & & & & rs & rs & FE4 & 1.3 \\
\hline - & & & & & & & & & ss & ss & FE4 & 0.4 \\
\hline G2 & 119/121 & $157 / 163$ & $125 / 135$ & 196/198 & $158 / 168$ & $115 / 115$ & $127 / 127$ & $121 / 127$ & ss & ss & FE4 & 4.6 \\
\hline G3 & $121 / 123$ & $155 / 163$ & $125 / 135$ & 196/196 & $168 / 168$ & $115 / 115$ & $131 / 131$ & $119 / 125$ & ss & ss & E4 & 3.8 \\
\hline G4 & $121 / 123$ & $144 / 163$ & $125 / 135$ & $196 / 198$ & $168 / 170$ & $115 / 115$ & $131 / 131$ & $127 / 127$ & rs & ss & FE4 & 0.4 \\
\hline - & & & & & & & & & $\mathrm{rr}$ & ss & FE4 & 0.4 \\
\hline G5 & 119/119 & $157 / 163$ & $125 / 127$ & 196/196 & $168 / 168$ & $115 / 115$ & $131 / 133$ & $121 / 121$ & ss & ss & FE4 & 0.4 \\
\hline G6 & 119/119 & $157 / 163$ & $125 / 127$ & 196/196 & $168 / 168$ & $115 / 115$ & 131/131 & $121 / 121$ & ss & ss & FE4 & 0.4 \\
\hline G7 & $121 / 123$ & 152/161 & $131 / 135$ & 198/198 & $158 / 168$ & $115 / 119$ & $131 / 137$ & $119 / 127$ & ss & ss & E4 & 0.4 \\
\hline G8 & $142 / 142$ & $159 / 163$ & $114 / 135$ & 196/198 & $168 / 170$ & $115 / 115$ & $127 / 127$ & $127 / 127$ & ss & ss & FE4 & 0.4 \\
\hline G9 & $115 / 144$ & $163 / 163$ & $125 / 125$ & 198/198 & $168 / 168$ & $115 / 115$ & $127 / 127$ & $127 / 127$ & ss & ss & $\mathrm{m}$ & 0.4 \\
\hline G10 & $119 / 144$ & $163 / 163$ & $125 / 125$ & 196/198 & $168 / 168$ & 115/115 & $127 / 127$ & $127 / 127$ & ss & ss & FE4 & 0.4 \\
\hline G11 & $121 / 126$ & $154 / 163$ & $125 / 129$ & 196/196 & $168 / 168$ & $115 / 117$ & $125 / 125$ & $137 / 137$ & ss & ss & E4 & 0.4 \\
\hline G12 & 119/119 & $157 / 163$ & $129 / 129$ & 196/196 & $168 / 170$ & $115 / 115$ & $129 / 129$ & $127 / 127$ & ss & rs & FE4 & 0.4 \\
\hline G13 & $121 / 127$ & $155 / 157$ & $129 / 135$ & 196/196 & $168 / 168$ & $115 / 115$ & $127 / 131$ & $127 / 127$ & ss & ss & FE4 & 0.4 \\
\hline G14 & 119/119 & $155 / 163$ & $125 / 125$ & 196/196 & $168 / 168$ & $115 / 115$ & $125 / 125$ & $119 / 127$ & $\mathrm{rr}$ & ss & FE4 & 0.4 \\
\hline G15 & 119/121 & $163 / 163$ & $125 / 135$ & 196/198 & $164 / 168$ & 115/115 & $129 / 129$ & $119 / 129$ & $\mathrm{rr}$ & rs & FE4 & 0.4 \\
\hline G16 & 119/121 & $157 / 163$ & $125 / 135$ & 196/198 & $164 / 168$ & $115 / 115$ & $127 / 127$ & $121 / 127$ & ss & ss & FE4 & 0.4 \\
\hline
\end{tabular}

'Gi' are the microsatellite multilocus genotypes (MLGs). The names of the MLGs and their microsatellite genotypes are omitted when they are the same than in the line above. The column 'Est. allele' refers to the alleles of the elevated esterases. 'freq' is the frequency of the type of individual in \% to one decimal place. Only the 237 individuals without missing data or with missing data (' $\mathrm{m}$ ' for G9) that do not influence the classification are shown (out of a total of 255 individuals).

gately parthenogenetic clone. This event could then have been followed by several years of selection and migration between populations without sexual recombination.

Under this hypothesis, G1 aphids should be obligately or partially asexual, that is, each winter at least part of the G1 lineage would be expected to reproduce by continuous parthenogenesis. On the other hand, the 15 rare MLGs detected in oilseed rape fields are not close relatives of G1, suggesting that none of the rare genotypes observed in this study have been recently derived from G1 by mutation. Although continuous parthenogenesis seems most likely to explain the frequency and geographic distribution of G1 individuals, the rare MLGs could have been sexually produced during the preceding winter.

Biological characterization of reproductive modes of various $M$. persicae genotypes would help evaluate the alternative hypotheses. To this end, the G1 and other genotypes could be subjected to conditions that induce sexual reproduction (ie low temperature and shortened day length).

\section{Insecticide resistance variation within and between $M$. persicae genotypes}

We found that genetic variation in insecticide resistance genotypes was present within microsatellite MLGs and that the same insecticide resistance mechanisms were present in different MLGs. We propose several hypotheses that could explain these unexpected results.

(1) Sexual recombination. The same resistance mutations in different MLGs could be readily explained if those genotypes have been generated by sexual reproduction. Recombination could also lead to a 
polymorphism at resistance genes within MLGs provided that multiple individuals with the same microsatellite genotypes were produced sexually. We have already argued that the probability of such outcomes is extremely small but could be higher because of the effects of selection.

(2) Cryptic meiotic or mitotic recombination. Different combinations of insecticide resistance mechanisms in individuals of the same clonal lineage could be the consequence of recombination localized in regions of the genome containing the insecticide resistance genes. Localized crossing over between homologues, or sister chromatid exchange, has previously been proposed to explain the distribution of rDNA arrays on X chromosomes in M. persicae (Hales et al, 2002). A careful analysis of insecticide resistance inheritance within parthenogenetic lines could allow this hypothesis to be tested.

(3) Polymorphism due to recent mutations. Resistance mutations at insecticide resistance genes could have occurred very recently, resulting in a transient resistance polymorphism within clonal MLGs. During this transition, both susceptible and resistant individuals would coexist within a MLG before the fixation of resistant genotypes. A study of temporal stability over several years is necessary to test this hypothesis. Also, mutations at microsatellite loci may explain the presence of the same insecticide resistance combination among different MLGs. Indeed, with more than 300 generations since the use of pyrethroids and organophophate insecticides, a large number of mutations at the microsatellite loci could have occurred within insecticide resistant clonal lineages.

(4) Multiple origins of insecticide resistance. Resistance mutations at each resistance gene could have appeared independently in different clonal MLGs. This could have been achieved by independent origins of the same mutation or by different mutations leading to the same resistant phenotype (homoplasy for the AChE for which the test is phenotypic). Multiple independent origins of the same insecticide resistance mutation within a species have already been reported in several cases (eg Andreev et al, 1999). The analysis of nucleotide variability surrounding the different insecticide resistance mutations should allow discrimination between single and multiple origins of each resistance mechanism.

\section{Selection vs genetic drift}

Only 16 microsatellite MLGs were detected among 255 M. persicae individuals collected from oilseed rape fields over a large geographic range in France (about $500 \mathrm{~km} \times 500 \mathrm{~km}$ ). This confirms the tendency for low genotypic variability in parthenogenetic populations of aphids, as observed in previous studies on $M$. persicae (Terradot et al, 1999; Guillemaud et al, 2003b; Vorburger et al, 2003) and on other species (eg Fuller et al, 1999; Simon et al, 1999; Delmotte et al, 2002; Papura et al, 2003).

However, the variability found in our study is far lower than that found in three previous studies that dealt with clonal populations of $M$. persicae collected at the regional scale. Fenton et al (1998) found 80 different genotypes among 276 aphids collected from potato and brassicas in 1995 in Scotland (in their study, the ratio of the number of genotypes over the number of individuals is $G=0.29$ ). An analysis of microsatellite diversity in samples collected from four aerial suction traps located in France in 2000 revealed higher variability, with 100 different MLGs among 174 flying individuals $(G=0.57)$ (Guillemaud et al, 2003b). In Victoria, Australia Vorburger et al (2003), detected 73 different MLGs among 167 individuals $(G=0.44)$ sampled on herbaceous hosts in 2002. We found only one frequent (G1) and 15 rare MLGs $(G=0.06)$. G1 was frequent in all the populations analyzed, whatever their location over a large geographic area (northern half of France), which is similar in size to the studies cited above. None of the frequent MLGs that were detected among flying aphids caught in aerial suction traps in France in 2000 (Guillemaud et al, $2003 \mathrm{~b}$ ) nor the frequent p6 MLG observed by Terradot et al (1999) among French and Spanish samples of $M$. persicae were the same as G1.

Founder events initiated by a few aphids followed by clonal reproduction could account for the very low genetic variability within populations. However, the ubiquitous distribution of G1 suggests that the samples considered in our study suffered a large reduction of genetic diversity due to a deterministic rather than a stochastic factor. A substantial migration could also homogenize the genotypic composition of the populations but it could not account for both the high frequency of G1 in oilseed rape fields and its apparent absence on other plants or in suction traps. In other words, we propose that genetic drift (or migration-drift balance) and parthenogenetic reproduction alone cannot account for the low variability observed. Large-scale selection probably accounts for this result.

\section{Does insecticide selection account for the low genetic variability of $M$. persicae populations?}

In France, oilseed rape fields are commonly treated with pyrethroid insecticides (Delorme et al, 1999); therefore, insecticide selection could be one of the primary causes of the reduced genetic variability. The combination of an elevated esterase (E4 or FE4) and the $k d r$ mutation, even in the heterozygous state, confers very high levels of resistance (Foster and Devonshire, 1999; Martinez-Torres et al, 1999; Foster et al, 2002).

In the samples analyzed here, the $k d r$ mutation was frequently associated with elevated esterases (90\% of the aphids) and these resistance mechanisms were combined in the most frequent microsatellite MLG, G1. Thus, these data support the hypothesis of an insecticide-induced selective sweep that reduced genetic variability in French populations of $M$. persicae feeding on oilseed rape.

However, the comparison of insecticide resistance mechanism frequencies between untreated fields and those treated with pyrethroids did not give clear-cut results. Indeed, most differences were not significant and some unexpected differences were detected. More $k d s / k d s$ individuals were found in a treated population (SG_2) than in two untreated fields. It is noteworthy that all $k d s / k d s$ individuals found in the treated sample SG_2 had the second most frequent microsatellite $\mathrm{MLG}^{-}$(G3), whereas the $k d r / k d s$ individuals had the genotype G1. It is therefore possible that the G3 genotype had a selective 
advantage over G1 (ie an undetected insecticide resistance mechanism). The super- $k d r$ mutation of the paralike gene, which confers resistance to pyrethroids in several insect species (Martinez-Torres et al, 1997), has been recently discovered in $M$. persicae (M. Williamson, IACR, Rothamsted, UK, 2003, personal communication), and is a plausible candidate.

\section{Multiple selection acting on populations of $M$. persicae}

The hypothesis of an insecticide-induced selective sweep is tempting. However, a few rare MLGs were found in association with both the $k d r$ mutation and an overproduced esterase. These rare genotypes and the frequent insecticide-resistant G1 were probably selectively equivalent in the face of the pesticide treatments used in these fields. Moreover, Guillemaud et al (2003a) found numerous (about 50) different MLGs that had the $k d r$ mutation and the overproduced esterase FE4 in sexually reproducing populations of $M$. persicae in France. These previous results, together with the present study, imply that insecticide selection alone cannot fully explain the observed genetic structure, in particular the high frequency of G1 in all populations (unless G1 possesses an additional undetected resistance gene, but see below).

A possible scenario involves two or more different selective pressures. The additional selective factor(s) may have favored one (or a few) particular genotype(s) in all oilseed rape fields sampled in 2001, but not the flying aphids caught in 2000 (Guillemaud et al, 2003b) nor aphids sampled on other plants in various years (Terradot et al, 1999) in the same region. We therefore propose that the additional selective pressure is a consequence of the agricultural practices associated with the oilseed rape cultivation and may include the nature of host plant itself. Host plant adaptation strongly affects the ecology and the genetic structure of many aphid species such as the cotton aphid Aphis gossypii (Glover) (Vanlerberghe-Masutti and Chavigny, 1998; Fuller et al, 1999) and the pea aphid Acyrthosiphon pisum (Via et al, 2000). Host plant specialization of adapted genotypes is theoretically possible in $M$. persicae; however, clear evidence of host-based genetic differentiation is lacking (Fenton et al (1998), but see Margaritopoulos et al (1998)). This evidence is not direct, as none of these studies considered aphids sampled from oilseed rape.

The alternative explanation for the predominance of G1 is that it possesses an additional undetected insecticide resistance gene. Although not impossible, this hypothesis seems unlikely because it could not explain why G1 was not detected among aphids caught outside oilseed rape fields (Terradot et al, 1999; Guillemaud et al, 2003b).

The present study suggests that human-induced selective pressures probably shape the genetic structure of $M$. persicae populations living on oilseed rape. Additional information is needed to evaluate fully the hypothesis that this selection involves both insecticide treatment and additional factors. In particular, the stability of the genetic structure over several years would provide strong support. In addition, genetic comparison of aphid populations growing on oilseed rape and those on other crops would help to ascertain the existence of host-adapted genotypes or host races.

\section{Acknowledgements}

We are grateful to HD Loxdale, N Miller, X Fauvergue, and D Forcioli for their advice and comments during this work and two anonymous reviewers for improvement of the text. We thank C Abba, E Buschiazzo, M Salles and $\mathrm{O}$ Migliore for their technical support in the laboratory, C Vorburger, P Sunnucks and A Wilson for their help with the microsatellite work, and the 'So What' association for its intellectual support. This study was funded by the 'Santé des Plantes et Environnement' Department of the 'Institut National de la Recherche Agronomique'.

\section{References}

Andreev D, Kreitman M, Phillips TW, Beeman RW, ffrench Constant RH (1999). Multiple origins of cyclodiene insecticide resistance in Tribolium castaneum (Coleoptera: Tenebrionidae). I Mol Evol 48: 615-624.

Anthony N, Unruh T, Ganser D, ffrench-Constant R (1998). Duplication of the $R d l$ GABA receptor subunit gene in an insecticide-resistant aphid, Myzus persicae. Mol Gen Genet 260: 165-175.

Balloux F, Lehmann L, de Meeus T (2003). The population genetics of clonal and partially clonal diploids. Genetics 164: 1635-1644.

Bengtsson BO (2003). Genetic variation in organisms with sexual and asexual reproduction. J Evol Biol 16: 189-199.

Blackman RL (1972). The inheritance of life-cycle differences in Myzus persicae (Sulz.) (Hem., Aphididae). Bull Entomol Res 62: 281-294.

Blackman RL, Spence JM, Field LM, Devonshire AL (1995). Chromosomal location of the amplified esterase genes conferring resistance to insecticides in Myzus persicae (Homoptera: Aphididae). Heredity 75: 297-302.

Daborn PJ, Yen JL, Bogwitz MR, Le Goff G, Feil E, Jeffers S et al (2002). A single P450 allele associated with insecticide resistance in Drosophila. Science 297: 2253-2256.

Delmotte F, Leterme N, Gauthier JP, Rispe C, Simon JC (2002). Genetic architecture of sexual and asexual populations of the aphid Rhopalosiphum padi based on allozyme and microsatellite markers. Mol Ecol 11: 711-723.

Delorme R, Ayala V, Auge D, Touton P, Ballanger Y (1999). Résistance du puceron vert du pêcher (Myzus persicae) aux insecticides dans le contexte de la culture du colza. 5e Conférence Internationale sur les Ravageurs en Agriculture, Montpellier, France, pp 81-89.

Devonshire AL, Devine GJ, Moores GD (1992). Comparison of microplate esterase assays and immunoassay for identifying insecticide resistant variants of Myzus persicae (Homoptera: Aphididae). Bull Entomol Res 82: 459-463.

Devonshire AL, Field LM (1991). Gene amplification and insecticide resistance. Annu Rev Entomol 36: 1-23.

Devonshire AL, Sawicki RM (1979). Insecticide-resistant Myzus persicae as an example of evolution by gene duplication. Nature 280: 140-141.

Fenton B, Woodford JA, Malloch G (1998). Analysis of clonal diversity of the peach-potato aphid, Myzus persicae (Sulzer), in Scotland, UK and evidence for the existence of a predominant clone. Mol Ecol 7: 1475-1487.

Field LM, Crick SE, Devonshire AL (1996). Polymerase chain reaction-based identification of insecticide resistance genes and DNA methylation in the aphid Myzus persicae (Sulzer). Insect Mol Biol 5: 197-202.

Foster SP, Denholm I, Devonshire AL (2002). Field-simulator studies of insecticide resistance to dimethylcarbamates and pyrethroids conferred by metabolic- and target sitebased mechanisms in peach-potato aphids, Myzus persicae (Hemiptera: Aphididae). Pest Manage Sci 58: 811-816. 
Foster SP, Devonshire AL (1999). Field-simulator study of insecticide resistance conferred by esterase-, MACE- and kdr-based mechanisms in the peach-potato aphid, Myzus persicae (Sulzer). Pest Sci 55: 810-814.

Fuller SJ, Chavigny P, Lapchin L, Vanlerberghe-Masutti F (1999). Variation in clonal diversity in glasshouse infestations of the aphid, Aphis gossypii Glover in southern France. Mol Ecol 8: 1867-1877.

Guillemaud T, Brun A, Anthony N, Sauge M-H, Boll R, Delorme $\mathrm{R}$ et al (2003a). Incidence of insecticide resistance alleles in sexually-reproducing populations of the peach-potato aphid Myzus persicae (Hemiptera: Aphididae) from southern France. Bull Entomol Res 93: 289-297.

Guillemaud T, Mieuzet L, Simon J-C (2003b). Spatial and temporal genetic variability in French populations of the peach-potato aphid, Myzus persicae. Heredity 91: 143-152.

Guillemaud T, Rooker S, Pasteur N, Raymond M (1996). Testing the unique amplification event and the worldwide migration hypothesis of insecticide resistance genes with sequence data. Heredity 77: 535-543.

Hales DF, Wilson ACC, Sloane MA, Simon J-C, Legallic J-F, Sunnucks P (2002). Lack of detectable genetic recombination on the $X$ chromosome during the parthenogenetic production of female and male aphids. Genet Res 79: 203-209.

Margaritopoulos JT, Mamuris Z, Tsitsipis JA (1998). Attempted discrimination of Myzus persicae and Myzus nicotianae (Homoptera: Aphididae) by random amplified polymorphic DNA polymerase chain reaction method. Ann Entomol Soc Am 91: 602-607.

Martinez-Torres D, Devonshire AL, Williamson MS (1997). Molecular studies of knockdown resistance to pyrethroids: cloning of domain II sodium chanel gene sequences from insects. Pest Sci 51: 265-270.

Martinez-Torres D, Foster SP, Field LM, Devonshire AL, Williamson MS (1999). A sodium channel point mutation is associated with resistance to DDT and pyrethroid insecticides in the peach-potato aphid, Myzus persicae (Sulzer) (Hemiptera:Aphididae). Insect Mol Biol 8: 339-346.

Moores GD, Devine GJ, Devonshire AL (1994). Insecticideinsensitive acetylcholinesterase can enhace esterase-based resistance in Myzus persicae and Myzus nicotianae. Pestic Biochem Physiol 49: 114-120.

Moores GD, Devonshire AL (2000). A fluorometric method to detect insensitive acetycholinesterase in resistant pests. Brighton Crop Protection Conference - Pests and Diseases, London 2000. BCPC, pp 447-452.

Papura D, Simon JC, Halkett F, Delmotte F, Le Gallic JF, Dedryver CA (2003). Predominance of sexual reproduction in, Romanian populations of the aphid Sitobion avenae inferred from phenotypic and genetic structure. Heredity 90: 397-404.

Raymond M, Rousset F (1995a). An exact test for population differentiation. Evolution 49: 1280-1283.

Raymond M, Rousset F (1995b). Genepop (version. 1.2), a population genetics software for exact tests and ecumenicism. J Hered 86: 248-249.
Rousset F (2001). Inferences from spatial population genetics. In: Balding DJ, Bishop M, Cannings C (eds) Handbook of Statistical Genetics. John Wiley \& Sons: Baffins Lanes. pp 239269.

Simon JC, Baumann S, Sunnucks P, Hebert PD, Pierre JS, Le Gallic JF et al (1999). Reproductive mode and population genetic structure of the cereal aphid Sitobion avenae studied using phenotypic and microsatellite markers. Mol Ecol 8: 531545.

Sloane M, Sunnucks P, Wilson ACC, Hales DF (2001). Microsatellite isolation, linkage group identification and determination of frequency in the peach-potato aphid, Myzus persicae (Sulzer) (Hemiptera: Aphididae). Genet Res 77: 251-260.

Sokal RR, Rohlf FJ (1995). Biometry. The Principles and Practice of Statistics in Biological Research, 3rd edn. W.H. Freeman and Company: New York.

Sommer SS, Groszbach AR, Bottema CDK (1992). PCR amplification of specific alleles (PASA) is a general method for rapidly detcting known single-base changes. BioTechniques 12: 82-87.

Sunnucks P, Hales DF (1996). Numerous transposed sequences of mitochondrial cytochrome oxidase I-II in aphids of the genus Sitobion (Hemiptera: Aphididae). Mol Biol Evol 13: 510524.

Terradot L, Simon J-C, Leterme N, Bourdin D, Wilson ACC, Gauthier J-P et al (1999). Molecular characterization of clones of the Myzus persicae complex (Hemiptera: Aphididae) differing in their ability to transmit the potato leafroll luteovirus (PLRV). Bull Entomol Res 89: 355-363.

Vanlerberghe-Masutti F, Chavigny P (1998). Host-based genetic differentiation in the aphid Aphis gossypii Glover, evidenced from RAPD fingerprints. Mol Ecol 7: 905-914.

Vanlerberghe-Masutti F, Chavigny P, Fuller SJ (1999). Characterization of microsatellite loci in the aphid species Aphis gossypii Glover. Mol Ecol 8: 693-695.

Via S, Bouck AC, Skillman S (2000). Reproductive isolation between divergent races of pea aphids on two hosts. II. Selection against migrants and hybrids in the parental environments. Evolution 54: 1626-1637.

Vorburger C, Lancaster M, Sunnucks P (2003). Environmentally related patterns of reproductive modes in the aphid Myzus persicae and the predominance of two 'superclones' in Victoria, Australia. Mol Ecol 12: 3493-3504.

Weir BS, Cockerham C (1984). Estimating F-statistics for the analysis of population structure. Evolution 38: 1358-1370.

Wilson ACC, Massonnet B, Simon JC, Prunier-Leterme N, Dolatti L, Llewellyn KS et al (2004). Cross-species amplification of microsatellite loci in aphids: assessment and application. Mol Ecol Notes 4: 104-109.

Wilson ACC, Sunnucks P, Blackman RL, Hales DF (2002). Microsatellite variation in cyclically parthenogenetic populations of Myzus persicae in south-eastern Australia. Heredity 88: 258-266.

Wright S (1969). Evolution and the Genetics of Populations. University of Chicago Press: Chicago, IL. 